\title{
Определение угарного газа металлоксидным сенсором
}

\author{
Чегерева К.Л., Шапошник А.В., Москалев П.В., Звягин А.А. \\ Воронежский государственный аграрный университет, Воронеж
}

Поступила в редакцию 30.09.2017 г.

\begin{abstract}
Селективное определение угарного газа выполнено с использованием единичного металлоксидного сенсора. Переход от стационарного нагрева полупроводникового сенсора к нестационарному позволил увеличить объем информации о газовой среде и провести селективный анализ. Обработка многомерных данных проводилась методом главных компонент.

Ключевые слова: нестационарный температурный режим, единичный металлоксидный сенcop, угарный газ, качественный анализ, количественный анализ, диоксид олова.
\end{abstract}

\section{Determination of carbon monoxide by the metal oxide sensor}

\author{
Chegereva K.L., Shaposhnik A.V., Moskalev P.V., Zvyagin A.A. \\ Voronezh State Agricultural University, Voronezh
}

To solve an important practical problem of determining the concentration of carbon monoxide in air, chemical sensors are usually used - semiconductor, electrochemical or thermocatalytic. Their main disadvantage is the low selectivity, which can lead to a false alarm when the analyzer enters the atmosphere of other reducing gases, for example, ethanol vapors, hydrogen, ammonia, etc. Increased selectivity was previously associated with the use of multisensory systems, the so-called "electronic noses", but later the researchers abandoned this idea. They found out that the instability of such devices increases with the increase in the number of sensors in a geometric progression. Currently, some researchers recognize the need to create devices for selective gas analysis based on a limited number of sensors - one or two.

For a reliable selective gas analysis, we need to obtain as much information about the gas medium as possible, which can not be done under stationary experimental conditions. The transition to nonstationary regimes increases the amount of information about the analyte because it reveals its features related to the chemisorption kinetics on the surface of the sensor, the kinetics of the chemical interaction between reductant analytes and chemisorbed oxygen, and the kinetics of desorption of chemical interaction products. This information is implicit in the data on the kinetics of the nonstationary process. Qualitative and quantitative analysis of gases using data on the kinetics of a nonstationary process requires the chemometric processing of multidimensional data.

Geometrically, each sample with a volume of $n$ resistance values can be represented as a point in $n$ dimensional space. In this case, the problem of identifying an analyte can be formulated as a cluster analysis problem, and the problem of determining the analyte concentration is a regression problem over a cloud of points belonging to the same cluster. However, the solution of these problems in the initial $n$-dimensional space is not very rational, since individual components of the vectors can be disorderly in information significance, and the range of their values can exceed three orders of magnitude. Statistical analysis of such data begins with the reduction of the dimension, for example, by the principal component method. To process the experimental data discussed in this paper, we used the package "chemometrics" developed by K. Varmuza and P. Filzmoser for a free system of statistical computations and graphics $R$.

Keywords: non-stationary temperature regime, single metal oxide sensor, carbon monoxide, qualitative analysis, quantitative analysis, tin dioxide. 


\section{Введение}

Угарный газ - это токсичное вещество, которое интенсивно образуется при работе на угольном или углеводородном топливе большинства видов тепловых двигателей и выделяется при пожарах. В выхлопных газах автомобилей примерно 1\% угарного газа. Угарный газ может связывать гемоглобин в крови, поэтому он является очень опасным веществом, предельно допустимая концентрация которого составляет всего 17 ppm. На оживленных автомобильных дорогах и в тоннелях концентрация угарного газа может превышать предельно допустимую в несколько раз.

Для того, чтобы избежать отравления, надо определять концентрацию угарного газа. Для этого могут быть использованы металлоксидные полупроводниковые сенсоры, предел обнаружения которых по угарному газу составляет примерно 0.1 ppm. Недостатком химических сенсоров является их низкая селективность.

В настоящее время применение простых в эксплуатации портативных газоанализаторов на основе химических сенсоров ограничивается их низкой селективностью. Ранее предполагалось, что задачи селективного анализа газов будут успешно решены с помощью мультисенсорных систем («электронных носов»). Однако выяснилось, что нестабильность работы подобных устройств существенно повышается при увеличении количества сенсоров. В связи с этим возникла необходимость создания устройств на основе ограниченного количества сенсоров.

Для селективного анализа газов необходимо получить достаточно большой объем информации об исследуемой газовой среде, что невозможно сделать при стационарных условиях эксперимента. Переход к нестационарным режимам увеличивает объем информации об аналите, потому что выявляет его особенности, связанные с кинетикой хемосорбции на поверхности сенсора, с кинетикой химического взаимодействия между аналитами-восстановителями и хемосорбированным кислородом, с кинетикой десорбции продуктов химического взаимодействия. Эти отличия в неявном виде содержатся в данных по кинетике нестационарного процесса. Для проведения качественного и количественного анализа необходимо проведение хемометрической обработки массивов многомерных данных с целью проведения качественного и количественного анализа.

\section{Эксперимент}

Для создания газочувствительного слоя металлоксидного сенсора использовали высокодисперсный порошок диоксида олова, полученного из оловянной кислоты. Для этого к раствору ацетата олова (+4) в ледяной уксусной кислоте добавляли избыток раствора аммиака:

$$
\mathrm{Sn}\left(\mathrm{CH}_{3} \mathrm{COO}\right)_{4}+4 \mathrm{NH}_{3}+3 \mathrm{H}_{2} \mathrm{O} \longrightarrow \mathrm{H}_{2} \mathrm{SnO}_{3} \downarrow+4 \mathrm{CH}_{3} \mathrm{COONH}_{4} \cdot \text { (1) }
$$

Образующуюся оловянную кислоту отделяли центрифугированием и промывали деионизованной водой, после чего просушивали, а затем прокаливали при температуре $450{ }^{\circ} \mathrm{C}$. В результате отжига формировался нанопорошок диоксида олова:

$$
\mathrm{H}_{2} \mathrm{SnO}_{3} \stackrel{t}{\longrightarrow} \mathrm{SnO}_{2}+\mathrm{H}_{2} \mathrm{O} .
$$

Для улучшения сенсорных свойств газочувствительного материала к нанодисперсному диоксиду олову добавляли катализаторы. В качестве допантов использовали нитрат тетраамминплатины (+2) и нитрат тетраамминпалладия (+2). Порошок с допантами смешивали с вязким наполнителем, раствором метилцеллозольва в амиловом спирте, для получения пасты, которую наносили на диэлектрическую подложку, содержащую платиновые электроды и платиновый 
нагреватель. Подложка с нанесенным на нее тонким слоем нагревалась до температуры $750^{\circ} \mathrm{C}$, в результате чего из пасты формировался высокодисперсный хрупкий гель. Платина и палладий, содержащиеся в комплексных соединениях, восстанавливались до металлов. После формирования газочувствительного слоя сенсор припаивался к корпусу.

На рис. 1 показан сенсорный отклик по отношению к угарному газу при стационарной температуре $433{ }^{\circ} \mathrm{C}$. Отклик сенсора (снижение электрического сопротивления) связан со взаимодействием угарного газа и хемосорбированных анионов кислорода:

$$
\mathrm{CO}_{2}+\mathrm{O}^{-} \stackrel{t}{\longrightarrow} \mathrm{CO}_{2}+\bar{e} .
$$

Аналогичные зависимости наблюдаются для других концентраций и для других газов-восстановителей, поэтому подобные исследования малопригодны для качественного анализа.

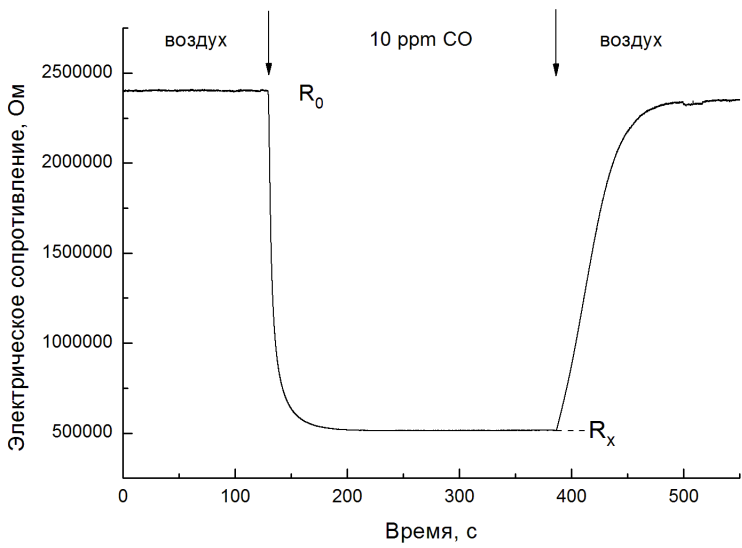

Рис. 1. Сопротивление сенсора на основе $\mathrm{SnO}_{2}$ с добавками палладия и платины при температуре $433^{\circ} \mathrm{C}$ при напуске 10 ppm CO.

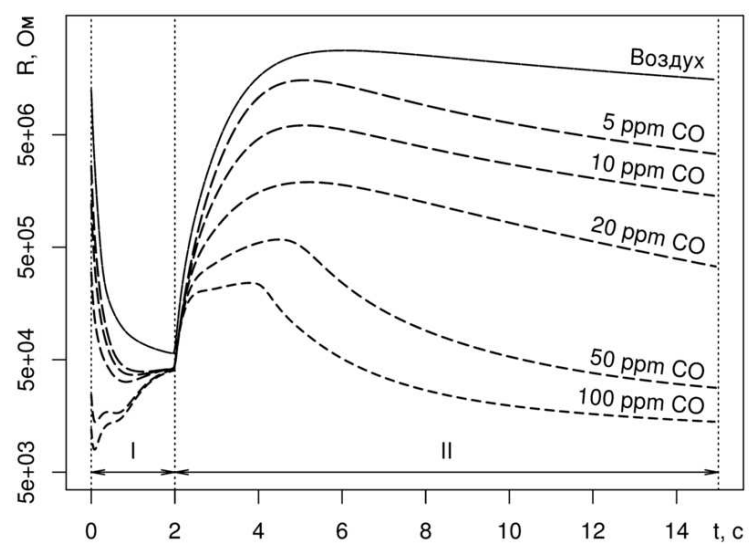

Рис. 2. Зависимость сопротивления сенсора от времени на протяжении одного цикла при нестационарном температурном режиме.

Кроме того, в наших исследованиях использовался нестационарный режим: нагрев до $450^{\circ} \mathrm{C}$ в течение 2 секунд (фаза I) и охлаждение до $100^{\circ} \mathrm{C}$ в течение 13 секунд (фаза II). Таким образом, один цикл измерений продолжался 15 секунд. Нестационарный температурный режим позволяет выявить природу газа-аналита. На рис. 2 показаны зависимости сопротивления сенсора на основе диоксида олова с добавками палладия и платины от времени в нестационарном режиме. На протяжении каждого цикла проводилось 750 измерений электрического сопротивления сенсора. Характер кривых при различных концентрациях угарного газа индивидуален для каждой концентрации аналита, показанной на рис. 2 длиной штрихов: самые длинные штрихи соответствуют 0 ppm CO, а самые короткие - 100 ppm CO. При этом нетрудно заметить, что для любого момента времени рост концентрации СО приводит к падению сопротивления сенсора.

\section{Обсуждение результатов}

С геометрической точки зрения каждая выборка объемом 750 значений сопротивления может быть представлена как точка в 750-мерном пространстве. В таком случае задача идентификации разновидности аналита может быть сформулирована как задача кластерного анализа, а задача определения концентрации аналита как задача регрессии по облаку точек, принадлежащих к одному кластеру. 
Однако непосредственное решение данной задачи в исходном 750-мерном пространстве не слишком рационально, поскольку его отдельные компоненты неупорядочены по информационной значимости, а размах их значений может превышать три порядка. В подобных условиях в статистическом анализе данных обычно используются методы редукции размерности, например, метод главных компонент [2].

Для обработки экспериментальных данных была использована библиотека «chemometrics», разработанная K. Varmuza и P. Filzmoser для свободной системы статистического анализа данных R [3]. Указанное программное обеспечение доступно на условиях свободной лицензии GNU GPL-3 на сайте CRAN [4]. На рис. 3 показаны результаты анализа полученных данных методом главных компонент.

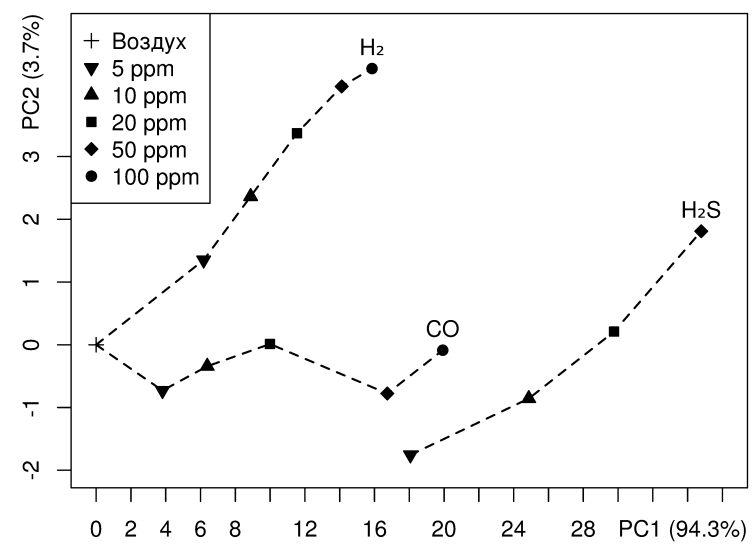

Рис. 3. Главные компоненты при определении угарного газа, водорода и сероводорода одиночным сенсором в нестационарном температурном режиме.

Нетрудно видеть, что линии, соответствующие трем из выбранных аналитов, не пересекаются, что предоставляет принципиальную возможность для качественного анализа газовой среды. Для проведения количественного анализа можно использовать как градуировочные зависимости, построенные по исходным значениям сопротивления сенсора, так и по первой паре главных компонент.

\section{Заключение}

Приведенные результаты показывают принципиальную возможность проведения качественного и количественного анализа на основе единичного малоселективного сенсора. Это может быть достигнуть за счет использования нестационарного температурного режима и последующей обработки многомерных данных с целью снижения их размерности.

\section{Список литературы}

1. Shaposhnik A., Zviagin A. et al. // Procedia Engineering. 2014. Vol. 87. pp. 1051-1054.

2. Буховец А.Г., Москалев П.В. Алгоритмы вычислительной статистики в системе R. СПб. Лань, 2015. 160 с.

3. Varmuza K., Filzmoser P. Introduction to Multivariate Statistical Analysis in Chemometrics. New York. CRC Press. 2009. 312 p.
4. Varmuza K., Filzmoser P. chemometrics: Multivariate Statistical Analysis in Chemometrics. Avialable at: https://cran.rproject.org/web/packages/chemometrics/index. html (accessed 29 September 2017). 


\section{References}

1. Shaposhnik A., Zviagin A. et al., Procedia Engineering, 2014, Vol. 87, pp. 1051-1054.

2. Buhovets A., Moskalev P., Algorithms of computational statistics in the system R. St. Petersburg, Lan, 2015. 160 p. (in Russian).

3. Varmuza K., Filzmoser P., Introduction to Multivariate Statistical Analysis in

Шапошник Алексей Владимирович д.х.н., заведующий кафедрой химии Воронежский аграрный университет им. императора Петра I, Воронеж.

Москалев Павел Валентинович - д.ф.-м.н. профессор кафедры математики и физики, Воронежский аграрный университет им. императора Петра I, Воронеж.

Звягин Алексей Алексеевич - доцент кафедры химии, Воронежский аграрный университет им.императора Петра I, Воронеж

Чегерева Кристина Леонидовна - аспирант кафедры химии Воронежский аграрный университет им.императора Петра I, Воронеж
Chemometrics. New York, CRC Press, 2009. $312 \mathrm{p}$.

4. Varmuza K., Filzmoser P. chemometrics: Multivariate Statistical Analysis in Chemometrics. Avialable at: https://cran.rproject.org/web/packages/chemometrics/index.h tml (accessed 29 September 2017).

Shaposhnik Alexey A. - head of chair of chemistry, Voronezh State Agrarian University, Voronezh, e-mail: a.v.shaposhnik@gmail.com

Moskalev Pavel V. - prof., grand Ph.D. (physics and mathematics), department of mathematics and physics, Voronezh State Agrarian University, Voronezh; e-mail: moskalefff@gmail.com

Zviagin Alexey A. - docent, chair of chemistry, Voronezh State Agrarian University, Voronezh.

Chegereva Kristina L. - postgraduate student, chair of chemistry, Voronezh State Agrarian University. Voronezh. 